\title{
Little Fish, Big Questions: A Collection of Modern Techniques for Mexican Tetra Research
}

Misty R. Riddle ${ }^{1}$, Clifford J. Tabin ${ }^{1}$

${ }^{1}$ Genetics Department, Blavatnik Institute, Harvard Medical School

\section{Corresponding Author}

Clifford J. Tabin

tabin@genetics.med.harvard.edu

\section{Date Published}

February 24,2020

\section{Editorial}

Despite a longstanding interest in the origins of animal diversity, we have a limited understanding of the genetic changes that underlie the evolution of morphology, physiology, and behavior. The Mexican tetra, Astyanax mexicanus, has emerged as a powerful model to further our understanding of how traits evolve. This species of fish exists as two morphotypes, an eyed river-dwelling morph (surface fish) that is abundant in rivers across Mexico and Southern Texas, and eyeless cave-dwelling morphs (cavefish) that thrive in the perpetually dark limestone caves within the Sierra del Abra region in Northeastern Mexico ${ }^{1}$. There are 29 known cave populations that have originated from at least two independent surface fish invasions ${ }^{2,3}$. The cave and river-morphs have remained interfertile and are easy to breed in the laboratory allowing researchers to genetically map the traits that evolve in response to the unique selective pressures in the cave. For example, absence of light has led to regression of eyes $4,5,6,7,8,9$, pigment $^{10,11,12,13,14}$, and sleep ${ }^{15,16,17,18,19}$; nutrient limitation has selected for overeating ${ }^{20}$, weight gain ${ }^{21}$, and fat storage ${ }^{22}$; and navigating in the dark has promoted increased ability to sense the environment ${ }^{23,24,25,26,27,28}$. A growing number

\section{Citation}

Riddle, M.R.,Tabin, C.J. Little Fish, Big Questions: A Collection of Modern Techniques for Mexican Tetra Research. J. Vis. Exp. (156), e60592, doi:10.3791/60592 (2020).
DOI

$10.3791 / 60592$
URL

jove.com/video/60592 of researchers are harnessing this fish to explore the genetic basis of evolution. Because some traits that are adaptive in cavefish, are detrimental in humans, the cavefish has also become attractive for biomedical research $21,29,30,31$. With the goal of promoting reproducibility and providing a platform for new investigators, this JoVE Methods Collection provides techniques for husbandry, trait measurement, and examination of gene activity.

As a starting point, the in vitro fertilization procedure demonstrated by Peuß et al. ${ }^{32}$ serves multiple purposes as it provides a method to obtain 1) cave/surface hybrids that may be difficult to breed, 2) one-cell stage embryos for microinjections, and 3) stage-matched samples for analysis of embryonic phenotypes. A. mexicanus typically spawn during the night. Peuß et al. describe a system to alter light-dark cycle and obtain fish that are primed for gamete collection during normal working hours. Their visual demonstration of gamete collection is particularly helpful as this step requires careful technique. Their article is essential for a number of downstream applications including comparison of gene expression patterns during embryogenesis. 
Luc et al. ${ }^{33}$ provide a robust in situ hybridization protocol optimized for visualizing gene expression in A. mexicanus embryos. They simplify this multi-day procedure using a checklist (provided as a supplementary file) and share guidelines for troubleshooting. Complementary to this method, Riddle et al. ${ }^{34}$ show how to examine gene expression and protein localization in hatched fish using wholemount immunohistochemistry. To make meaningful comparisons between morphotypes after hatching and feeding begin, it is necessary to control the growth conditions. They show how to breed adults through natural spawning and raise larvae at fixed densities on a rotifer diet that has considerable advantages in nutrient content and cost compared to other food sources. Their immunostaining protocol successfully recognizes antigens in the brain, gut, and pancreas as late as 12-days post hatching allowing late stages of organ development and function to be interrogated. Importantly, they provide a list of antibodies that have been successfully used in A. mexicanus. The combined methods of Luc et al. and Riddle et al. are useful to analyze the effects of genetic manipulation.

In this Methods Collection, Stahl et al. ${ }^{35}$ describe multiple ways to manipulate gene function in $A$. mexicanus including: morpholino microinjection, CRISPR-Cas9mediated mutagenesis, and Tol2-mediated transgenesis. In addition to demonstrating pico-injection and screening of injected embryos, they provide helpful guidelines for designing morpholinos, guide RNAs, and transgenes. They share results that validate each of these techniques. For example, targeting Hypocretin/orexin (HCRT) using a spliceblocking morpholino reduces locomotor activity and increases sleep in cavefish; editing oca2 (the causative locus for cavefish albinism) in surface fish using CRISPR/Cas9 produces albino surface fish; and inserting a genetically encoded calcium indicator driven by a pan-neuronal zebrafish promotor using Tol2 transgenesis achieves nearubiquitous neuronal expression in the Mexican tetra. They point out that many of the genetic tools developed in zebrafish can be directly transferred to cavefish because of their similar genomes. Their article is an essential resource for researchers performing functional studies in $A$. mexicanus and complements the additional articles focused on measuring phenotypes, including behavior.

Cavefish have dramatically reduced sleep and altered circadian rhythm $36,37,38$. This presents an exciting opportunity to investigate the contribution of genetics and environment to variation in sleep in a vertebrate. Jaggard et al. ${ }^{39}$ show how to build a system from inexpensive materials that can be used to record sleep at different life stages and under different contexts. Further, they describe how to automate fish tracking in this set-up and obtain quantitative measurements for sleep and locomotion. Their technique is applicable for high-throughput drug and genetic screening as well as traditional genetic mapping studies.

In addition to reduced sleep, cavefish have evolved differences in locomotor activities linked to foraging, including altered feeding angle and vibration attraction ${ }^{16,30,40}$. Central to understanding how these activities evolved is linking them to developmental changes. Worsham et al. ${ }^{41}$ present methods for recording behaviors and correlating them with changes in neuromasts; external mechanosensory structures that respond to water flow. They first show how to create chambers to record vibration attraction and sleep, and provide a detailed description of how to quantify the behaviors using free software and customizable scripts. Next, they demonstrate how to visualize neuromasts in living adult fish using a fluorescent mitochondrial stain and again, using free 
software, how to quantify the number of neuromasts in a semi-automated fashion. Vibration attraction and neuromast number are correlated ${ }^{23}$; their method provides a step by step protocol to interrogate this relationship. The methods of Jaggard and Worsham can be used to uncover additional behaviors and can also be applied to other species of fish. These methods produce quantitative and consistent results that are essential to discovering the genetic basis of behavioral evolution.

Modern techniques to measure and manipulate gene function have pushed evolutionary studies from comparative to mechanistic. Combined, the articles in this Methods Collection serve as a guidebook to carry out investigations of gene function in $A$. mexicanus. While researchers have uncovered fascinating differences in morphology, physiology, and behavior between surface and cave morphs, there are many traits that have yet to be explored in detail, and likely more to discover. As more work is done, this little fish will continue to have a big impact on our understanding of evolution.

\section{Disclosures}

The authors have nothing to disclose.

\section{Acknowledgments}

The authors acknowledge funding from the National Institutes of Health (NH089934, DK108495). F. Leal and A. Powers are acknowledged for editing.

\section{References}

1. Mitchell, R. W., Russel, W. H., Elliot, W. R. Mexican eyeless characin fishes, Genus Astyanax: Environment,
Distribution, and Evolution. Special Publications, the Museum Texas Tech University. 12, (1977).

2. Elliott, W. R. Cave Exploration and Mapping in the Sierra de El Abra Region. in Biology and Evolution of the Mexican Cavefish. 9-40 (2015).

3. Herman, A. et al. The role of gene flow in rapid and repeated evolution of cave-related traits in Mexican tetra, Astyanax mexicanus. Molecular Ecology. 27, 4397-4416 (2018).

4. Rohner, N. et al. Cryptic variation in morphological evolution: HSP90 as a capacitor for loss of eyes in cavefish. Science (80). 342, 1372-1375 (2013).

5. McGaugh, S. E. et al. The cavefish genome reveals candidate genes for eye loss. Nature Communications. 5, (2014).

6. Lyon, A., Powers, A. K., Gross, J. B., O'Quin, K. E. Two Three loci control scleral ossicle formation via epistasis in the cavefish astyanax mexicanus. PLoS One. 12, (2017).

7. Atukorala, A. D. S., Franz-Odendaal, T. A. Genetic linkage between altered tooth and eye development in lens-ablated Astyanax mexicanus. Developmental Biology. (2018).

8. Gore, A. V et al. An epigenetic mechanism for cavefish eye degeneration. Nature Ecology \& Evolution. 2, 1155-1160 (2018).

9. Sumner-Rooney, L. The kingdom of the blind: disentangling fundamental drivers in the evolution of eye loss. Integrative and Comparative Biology. (2018).

10. Protas, M. E. et al. Genetic analysis of cavefish reveals molecular convergence in the evolution of albinism. Nature Genetics. 38, 107-111 (2006). 
11. Protas, M., Conrad, M., Gross, J. B., Tabin, C., Borowsky, R. Regressive Evolution in the Mexican Cave Tetra, Astyanax mexicanus. Current Biology. 17, 452-454 (2007).

12. Protas, M. et al. Multi-trait evolution in a cave fish, Astyanax mexicanus. Evolution \& Development. 10, 196-209 (2008).

13. Stahl, B. A., Gross, J. B. Alterations in Mc1r gene expression are associated with regressive pigmentation in Astyanax cavefish. Development Genes and Evolution. 225, 367-375 (2015).

14. McCauley, D. W., Hixon, E., Jeffery, W. R. Evolution of pigment cell regression in the cavefish Astyanax: $A$ late step in melanogenesis. Evolution \& Development. 6 , 209-218 (2004).

15. Duboué, E. R., Keene, A. C., Borowsky, R. L. Evolutionary convergence on sleep loss in cavefish populations. Current Biology. 21, 671-676 (2011).

16. Yoshizawa, M. et al. Distinct genetic architecture underlies the emergence of sleep loss and prey-seeking behavior in the Mexican cavefish. BMC Biology. 13, 15 (2015).

17. Jaggard, J. et al. The lateral line confers evolutionarily derived sleep loss in the Mexican cavefish. Journal of Experimental Biology. 220, 284-293 (2017).

18. Jaggard, J. B. et al. Hypocretin underlies the evolution of sleep loss in the Mexican cavefish. Elife. 7, (2018).

19. Duboué, E. R., Borowsky, R. L., Keene, A. C. $\beta$ adrenergic signaling regulates evolutionarily derived sleep loss in the mexican cavefish. Brain, Behavior and Evolution. 80, 233-243 (2012).
20. Aspiras, A. C., Rohner, N., Martineau, B., Borowsky, R. L., Tabin, C. J. Melanocortin 4 receptor mutations contribute to the adaptation of cavefish to nutrientpoor conditions. Proceedings of the National Academy of Sciences of the United States of America. 112, 9668-9673 (2015).

21. Riddle, M. R. et al. Insulin resistance in cavefish as an adaptation to a nutrient-limited environment. Nature. 555, 647-651 (2018).

22. Xiong, S., Krishnan, J., Peuß, R., Rohner, N. Early adipogenesis contributes to excess fat accumulation in cave populations of Astyanax mexicanus. Developmental Biology. (2018).

23. Yoshizawa, M., Gorički, Š., Soares, D., Jeffery, W. R. Evolution of a behavioral shift mediated by superficial neuromasts helps cavefish find food in darkness. Current Biology. 20, 1631-1636 (2010).

24. Franz-Odendaal, T. A., Hall, B. K. Modularity and sense organs in the blind cavefish, Astyanax mexicanus. Evolution and Development. 8, 94-100 (2006).

25. Hinaux, $H$. et al. Sensory evolution in blind cavefish is driven by early embryonic events during gastrulation and neurulation. Development. 143, 4521-4532 (2016).

26. Blin, M. et al. Developmental evolution and developmental plasticity of the olfactory epithelium and olfactory skills in Mexican cavefish. Developmental Biology. (2018).

27. Lloyd, E. et al. Evolutionary shift towards lateral line dependent prey capture behavior in the blind Mexican cavefish. Developmental Biology. 441, 328-337 (2018).

28. Powers, A. K., Boggs, T. E., Gross, J. B. Canal neuromast position prefigures developmental patterning 
of the suborbital bone series in Astyanax caveand surface-dwelling fish. Developmental Biology. 441, 252-261 (2018).

29. Rohner, N. "Out of the Dark" Cavefish Are Entering Biomedical Research. in Zebrafish, Medaka, and Other Small Fishes. 253-268 (2018).

30. Yoshizawa, M. et al. The evolution of a series of behavioral traits is associated with autism-risk genes in cavefish. BMC Evolutionary Biology. 18, 89 (2018).

31. Stockdale, W. T. et al. Heart Regeneration in the Mexican Cavefish. Cell Reports. 25, 1997-2007.e7 (2018).

32. Peuß, R. et al. Gamete Collection and In Vitro Fertilization of Astyanax mexicanus. Journal of Visualized Experiments. (2019).

33. Luc, H., Sears, C., Raczka, A., Gross, J. B. Wholemount In Situ Hybridization for Astyanax Embryos. Journal of Visualized Experiments. (2019).

34. Riddle, M., Martineau, B., Peavey, M., Tabin, C. Raising the Mexican Tetra Astyanax mexicanus for Analysis of Post-larval Phenotypes and Wholemount Immunohistochemistry. Journal of Visualized Experiments. (2018).

35. Stahl, B. A. et al. Manipulation of Gene Function in Mexican Cavefish. Journal of Visualized Experiments. (2019).

36. Duboué, E. R., Keene, A. C. Investigating the Evolution of Sleep in the Mexican Cavefish. in Biology and Evolution of the Mexican Cavefish. 291-308 (2015).

37. Frøland Steindal, I. A., Beale, A. D., Yamamoto, Y., Whitmore, D. Development of the Astyanax mexicanus circadian clock and non-visual light responses. Developmental Biology. (2018).
38. Carlson, B. M., Gross, J. B. Characterization and comparison of activity profiles exhibited by the cave and surface morphotypes of the blind Mexican tetra, Astyanax mexicanus. Comparative Biochemistry and Physiology Part C: Toxicology \& Pharmacology. 208, 114-129 (2018)

39. Jaggard, J. B., Lloyd, E., Lopatto, A., Duboue, E. R., Keene, A. C. Automated Measurements of Sleep and Locomotor Activity in Mexican Cavefish. Journal of Visualized Experiments. (2019).

40. Kowalko, J. E. et al. Convergence in feeding posture occurs through different genetic loci in independently evolved cave populations of Astyanax mexicanus. Proceedings of the National Academy of Sciences of the United States of America. 110, 16933-16938 (2013).

41. Worsham, M. et al. Behavioral Tracking and Neuromast Imaging of Mexican Cavefish. Journal of Visualized Experiments. (2019). 